\title{
VCP-dependent muscle degeneration is linked to defects in a dynamic tubular lysosomal network in vivo
}

\author{
Alyssa E Johnson, Huidy Shu, Anna G Hauswirth, Amy Tong, Graeme W Davis* \\ Department of Biochemistry and Biophysics, University of California, San Francisco, \\ San Francisco, United States
}

\begin{abstract}
Lysosomes are classically viewed as vesicular structures to which cargos are delivered for degradation. Here, we identify a network of dynamic, tubular lysosomes that extends throughout Drosophila muscle, in vivo. Live imaging reveals that autophagosomes merge with tubular lysosomes and that lysosomal membranes undergo extension, retraction, fusion and fission. The dynamics and integrity of this tubular lysosomal network requires VCP, an AAA-ATPase that, when mutated, causes degenerative diseases of muscle, bone and neurons. We show that human VCP rescues the defects caused by loss of Drosophila VCP and overexpression of disease relevant VCP transgenes dismantles tubular lysosomes, linking tubular lysosome dysfunction to human VCP-related diseases. Finally, disruption of tubular lysosomes correlates with impaired autophagosome-lysosome fusion, increased cytoplasmic poly-ubiquitin aggregates, lipofuscin material, damaged mitochondria and impaired muscle function. We propose that VCP sustains sarcoplasmic proteostasis, in part, by controlling the integrity of a dynamic tubular lysosomal network.
\end{abstract}

DOI: 10.7554/eLife.07366.001

*For correspondence: graeme. davis@ucsf.edu

Competing interests:

See page 18

Funding: See page 18

Received: 07 March 2015

Accepted: 11 July 2015

Published: 13 July 2015

Reviewing editor: $\mathrm{K}$ VijayRaghavan, National Centre for Biological Sciences, Tata Institute for Fundamental Research, India

Copyright Johnson et al. This article is distributed under the terms of the Creative Commons Attribution License, which permits unrestricted use and redistribution provided that the original author and source are credited.

\section{Introduction}

Valosin-containing protein (VCP), the homologue of yeast Cdc48, is the causative gene for a multisystem degenerative disease that was originally termed IBMPFD to encompass the wide range of debilitating clinical outcomes, including inclusion body myopathy (IBM), Paget's disease of the bone (PDB) and frontotemporal dementia (FD) (Watts et al., 2004). Recently, the list of degenerative disorders that are associated with VCP mutations has expanded to include amyotrophic lateral sclerosis (ALS) (Abramzon et al., 2012), spastic paraplegia (Clemen et al., 2010), scapuloperoneal muscular dystrophy (Liewluck et al., 2014) and Charcot-Marie-Tooth disease (Gonzalez et alo, 2014). Currently, there are no viable treatments available to slow or halt progression of VCP-related diseases.

Muscle weakness is the first presenting symptom in over 50\% of VCP disease patients (Weihl et al., 2009), yet very little is known about the muscle pathology of VCP-related diseases. Muscle biopsies from patients with VCP-related diseases display an accumulation of cytoplasmic poly-ubiquitin aggregates (Watts et al., 2004; Weihl et al., 2009; Dolan et al., 2011), suggesting a major defect in protein clearance. VCP is an AAA-ATPase that has essential functions in ubiquitin-dependent proteolysis. But, pathogenic mutations in VCP do not seem to impair the UPS or ERAD protein degradation pathways (Tresse et al., 2010a; Chang et al., 2011). More recently, VCP has been implicated in autophagy. Specifically, over-expression of VCP mutant transgenes with disease causing mutations leads to an accumulation of autophagosomes (Ju et al., 2009; Tresse et alo, 2010a), suggesting that VCP functions in processes related to the maturation or fusion of autophagosomes with lysosomes.

Lysosomes are the major cellular degradation sites for clearing damaged proteins and organelles. Lysosomes are classically thought to be vesicular organelles, where they serve as depots for cargo 
eLife digest Mutations in a gene that produces a protein called Valosin-containing protein (VCP for short) causes degenerative diseases that affect the brain, muscle and bone. In nearly half of the individuals with these VCP-related diseases-which can also result in dementia, Paget's disease of the bone and amyotrophic lateral sclerosis (ALS)—-the first symptom is muscle weakness. Currently, very little is known about how VCP affects muscles.

Patients with VCP-related diseases often have problems clearing damaged proteins from their cells, and recent research suggests that VCP is important for forming a cellular structure known as a lysosome. Lysosomes contain powerful enzymes that destroy damaged proteins and other cellular structures that would otherwise accumulate in the cells. In most cells, lysosomes look like bubble-like compartments called vesicles. However, in some types of cells lysosomes have been observed to form a network of tubules that extend throughout the cell interior. However, it remains unclear what these tubules do, how they form in cells and whether they are altered in disease.

Johnson et al. analyzed lysosomes in the muscle of the fruit fly species Drosophila melanogaster and discovered that lysosomes were in the form of a network of tubules that spread throughout each muscle cell. These tubules constantly changed in living muscles; extending, retracting, breaking and merging to form a large tubular lysosome network. When Johnson et al. reduced the amount of VCP produced by the muscle cells, via a method called RNA interference, the lysosome tubules broke down into vesicles that were no longer constantly changing. Modifying these defective fly muscle cells so that they produced the human VCP protein caused the tubules to form again. These results suggest that the human and fly VCP proteins are very similar and that they play a key role in either the ability of lysosomes to form tubules or the maintenance of existing tubules.

Johnson et al. then engineered flies to produce a version of the VCP protein that had mutations commonly seen in individuals with degenerative diseases. Lysosome tubules did not form correctly in the muscle cells of these flies. These flies also had other abnormalities; for example, their cells showed a great build-up of damaged proteins, and their ability to move their muscles was weaker.

These findings suggest that a network of lysosomal tubules is necessary for healthy muscle cells, but how and why these tubular networks are formed or maintained is still mysterious. What causes lysosomal membranes to form tubules? How do they break and fuse? And why are they necessary? Genetic experiments in fruit flies will be a great place to discover these mechanisms and understand the links to degenerative diseases in humans.

DOI: 10.7554/eLife.07366.002

delivered via endosomes or autophagosomes. However, in certain systems lysosomes have been observed to adopt non-vesicular morphologies. A particularly dramatic example has been observed in a subset of bone-derived cultured macrophages, where lysosomes form abundant, extended tubules that radiate from the cell center and, in some cases, form an interconnected web throughout the cytoplasm (Swanson et alo, 1987a; Knapp and Swanson, 1990). Additionally, there are examples where cellular stress, particularly the induction of high levels of autophagy, induces lysosomal membranes to tubulate and undergo scission to produce new vesicular lysosomes, a process referred to as autophagic lysosome reformation (ALR) (Yu et alo, 2010). Despite these observations, lysosome tubules have received little attention and it remains unclear to what extent lysosome tubulation occurs in different cell types and what purpose it serves in vivo. Moreover, the molecular repertoire of factors required for lysosome tubule formation is virtually unknown.

Here, we employ fluorescently tagged lysosomal and autophagic markers to study the autophagylysosome system in Drosophila muscle cells and investigate the muscle pathology of VCP-related diseases. Remarkably, we find that lysosomes adopt a dynamic, tubular morphology that ramifies throughout the entire sarcoplasm of Drosophila muscle, in vivo. We find that VCP is required for the integrity and dynamics of this tubular network. Disruption of lysosome tubules correlates with defects in autophagosome-lysosome fusion, increased poly-ubiquitin aggregates and the accumulation of lipofuscin material in the sarcoplasm. We show that the human VCP homolog can rescue lysosomal tubulation following loss of VCP in Drosophila muscle, indicating that the functions of VCP in lysosome tubulation are conserved. Finally, we demonstrate that homologous mutations that cause VCP diseases in human patients disrupt the lysosome tubular lattice, suggesting that disruption of 
lysosome tubules contributes to VCP mutant pathogenesis. Taken together, our data establish a functional link between lysosome tubule dysfunction and the pathology of VCP-related degenerative diseases.

\section{Results}

\section{Drosophila sarcoplasmic lysosomes form an extended dynamic tubular array in vivo}

To visualize muscle lysosomes in vivo, we expressed RFP-tagged Spinster, which has previously been defined as a late endosomal/lysosomal transmembrane protein (Sweeney and Davis, 2002; Dermaut et al., 2005). Remarkably, when Spinster-RFP is expressed in Drosophila muscle, Spin-RFP localizes to an expansive tubular network (Figure $1 \mathrm{~A}-\mathrm{C}$ and Video 1). Tubules were found evenly distributed throughout the sarcoplasm and formed a web of connections with other tubules (Figure 1B,C). Tubules were observed in every muscle and there were no apparent differences in the tubule abundance or architecture between different muscles. We also observed enlarged vesicular compartments at tubule intersections throughout the muscle (Figure 1C). This network is highly sensitive to all chemical fixation conditions that we have attempted. When subjected to fixation, the tubule lattice collapses (Figure 1D), leaving behind distributed round, Spinster-positive compartments that resemble classically defined late-endosomal, lysosomal structures (Sweeney and Davis, 2002; Dermaut et al., 2005). Thus, live imaging is essential to study the function and relevance of this Spin-positive, tubular network.

We next explored the dynamics of this tubular network in time-lapse videos. We find evidence of tubule extension, retraction, scission and fusion (Figure 1E-G). The dominant activity in the network is the dynamic extension and retraction of individual tubules throughout the sarcoplasm (Figure 1E and Video 2). Often the same tubule was observed to extend and retract, while surrounding tubules remained constant. The impression is that the lattice has both stable, interconnected tubules and dynamic elements that create new connections or retract once a connection is broken. In less frequent instances, we observed scission events from the end of a tubule, resulting in a mobile vesicle (Figure 1F). In a corollary phenomenon, we observed tubule extensions that resulted in tubules fusing to larger nodes in the network (Figure 1G). Finally, we observed de novo formation of tubules extruding from the side of existing tubules (Figure 1G, last panel) demonstrating that tubulation can originate from existing tubules, not just pre-existing nodes. Importantly, the existence of a tubular network and tubule dynamics are not an artifact of a dissected neuromuscular preparation. We observed an identical, dynamic tubular lysosomal network in intact larvae that were imaged through the cuticle while restrained in a microfluidics chamber (Video 3).

The observed lysosomal network structure and dynamics are consistent with the involvement of either the actin or microtubule cytoskeletons. First, we tested whether the tubules require an intact microtubule cytoskeleton. Treatment with nocodazole for $1 \mathrm{hr}$ completely abolished tubules, indicating that the tubules require microtubule support (Figure $1 \mathrm{H}, \mathrm{l})$. In contrast, disruption of the actin cytoskeleton with latrunculin A did not have a significant effect on the tubular network, indicating a non-essential role for the actin cytoskeleton in maintaining lysosome tubules (Figure 1J). Finally, we tested whether Clathrin is essential for tubular network integrity. Clathrin has the capacity to shape membranes and was recently implicated in the process of ALR in cultured mammalian cells (Rong et al., 2012), a process that involves limited tubulation from auto-lysosomal compartments. Expression of Clathrin heavy chain RNAi (Chc-RNAi) completely disrupted network integrity (Figure 1K).

Although Spinster was characterized previously as a lysosomal marker, the tubular structures labeled by Spinster are dramatically different from the classical view of vesicular lysosomes. Therefore, we performed additional experiments to validate that the tubular network is lysosomal. First, we coimaged Spin-GFP with the low pH fluorescent probe Lysotracker and found complete co-localization (Figure 2A), indicating that the Spinster network is acidic. To verify that the observed tubular network is not an artifact of Spin-RFP over-expression, we stained wild type muscles with Lysotracker to examine lysosome morphology under wild type conditions. Lysotracker staining confirmed the existence of this tubular network in wild type muscle (Figure 2B). We also note that the tubule intersections stained more intensely for Lysotracker than the tubules themselves. This could be due to increased tubule volume at the intersections, or these sites might actually have a lower $\mathrm{pH}$ than the 

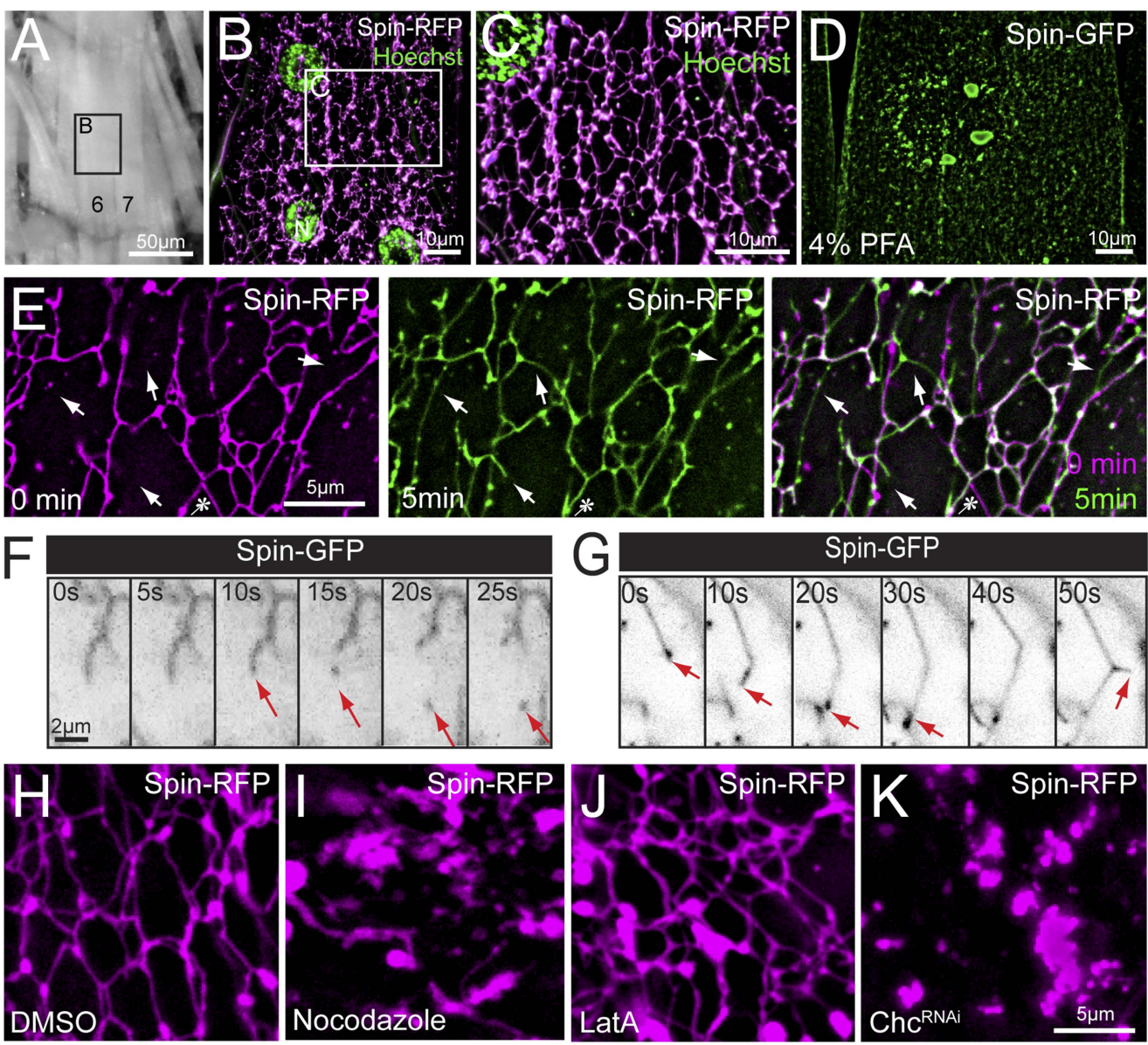

Figure 1. Lysosomes adopt an extended dynamic tubular array in Drosophila sarcoplasms. (A) Muscles of third instar larvae from segment A2. (B) Representative live image of Spin-RFP expressed in muscles at 63x magnification. Muscle 4 (B) is shown. DNA was stained with Hoescht. (C) Representative live image of Spin-RFP expressed in muscles using the muscle-specific MHC-Gal4 driver. DNA was stained with Hoescht. (D) Representative image of Spin-GFP localization in a muscle that was fixed with 4\% PFA prior to imaging. (E) Time-lapse images of the Spin-RFP network. Time 0 is represented in magenta, and the 5 min time-point is represented in green. The 2 time points were merged to show new and lost tubule formations over the course of $5 \mathrm{~min}$. Arrows indicate examples of de novo tubule formations and the asterisk indicates a retracted tubule. (F) Representative time-lapse sequence of a SpinGFP tubule fission event. (G) Representative time-lapse sequence of a Spin-GFP tubule fusion event. In the last frame, a de novo tubule can be seen extruding from the middle of a pre-existing tubule. $(\mathbf{H}-\mathbf{J})$ Spin-RFP localization in muscles treated with DMSO (H), Nocodazole (I) or LatA (J). (K) Spin-RFP localization in muscles expressing Clathrin heavy chain (Chc) RNAi.

DOI: 10.7554/eLife.07366.003

tubules themselves. Finally, we co-imaged Spin-RFP with several other organelle markers to verify that Spinster specifically labels lysosomes and does not co-stain other organelles. ER and mitochondria also form tubule structures, but when we co-imaged Spin-RFP with ER-tracker and Mito-tracker fluorescent dyes, Spin-RFP tubules did not co-localize with either ER or mitochondria tubules (Figure 2C,D). Instead, Spin-RFP tubules were interwoven between mitochondria and ER tubules. Additionally, Spin-RFP did not co-localize with markers for early endosomes (YFP-Rab5), recycling endosomes (Rab11-GFP), medial Golgi (Manll-GFP) or trans Golgi (GalT-YFP) (Figure 2E-H). We note that Golgi organization in muscles forms vesicular structures rather than the classical Golgi stacks that are observed in most cell types and this is consistent with Golgi organization that has been observed in vertebrate skeletal muscles (Ralston et al., 2001). Collectively, we have identified and characterized 


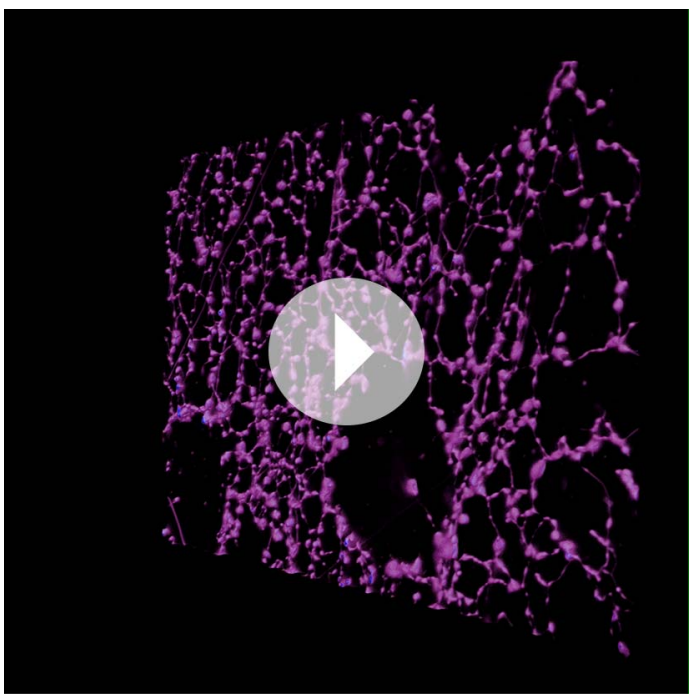

Video 1. Spin-RFP tubular network in Drosophila muscle. Spin-RFP was expressed in muscles and imaged live. Average Z-stacks were assembled to produce a 3D volume projection and various angles of the projections are shown.

DOI: 10.7554/eLife.07366.004

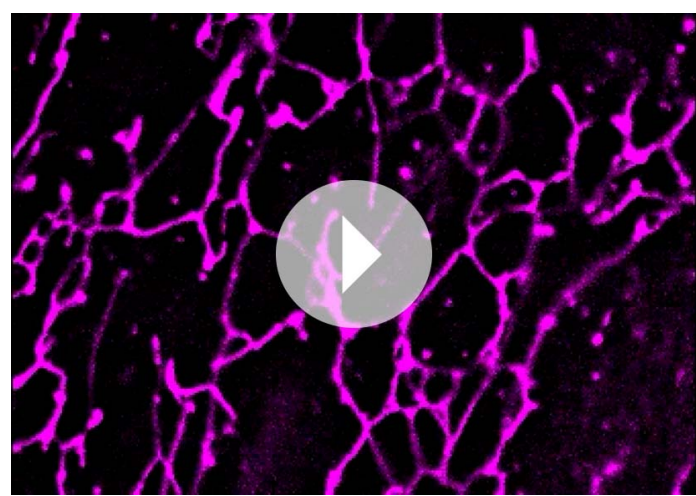

Video 2. Spin-RFP tubule dynamics in Drosophila muscle. Representative time-lapse video of Spin-RFP expressed in muscles. Frames were taken at $10 \mathrm{~s}$ intervals.

DOI: 10.7554/eLife.07366.005

a dynamic tubular lysosomal network that permeates the entire sarcoplasm of Drosophila body-wall muscle in vivo.

\section{VCP is required for the integrity and dynamics of the extended tubular lysosomal network}

To investigate the molecular underpinnings of the observed lysosomal tubule dynamics, we pursued a candidate-based RNAi screen to identify genes required for lysosome tubulation. We focused on genes that have been implicated in the autophagy-lysosome system and identified the AAA-ATPase VCP as being required for the integrity of the entire tubular lysosome network. Specifically, inhibiting VCP expression by RNAi abolished lysosome tubules, leaving behind vesicles throughout the sarcoplasm (Figure $3 A, B$ ). The lysosome vesicles were irregular in their size and shape and appeared clustered, rather than uniformly distributed throughout the sarcoplasm. To determine whether the catalytic ATPase function of VCP is required for the tubular network integrity, we employed a VCP-selective inhibitor $\mathrm{DBeQ}$ (Chou et al., 2011). Acute inhibition of VCP with DBeQ

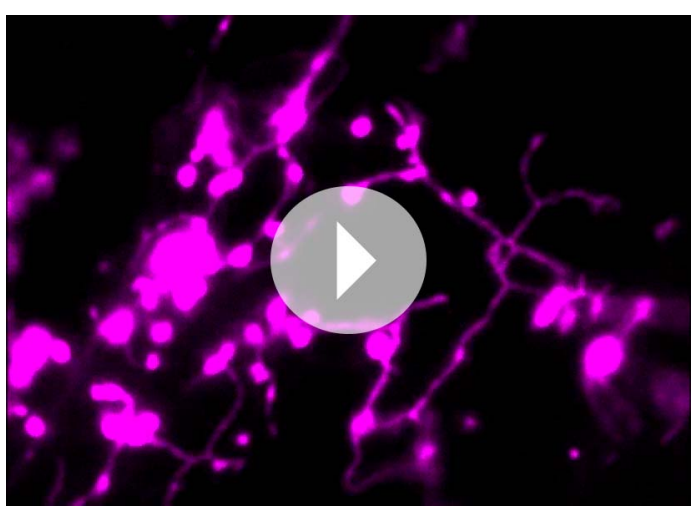

Video 3. Spin-RFP tubule dynamics in an intact larva. A whole un-dissected larva was immobilized in a mircrofluidics chamber and Spin-RFP was imaged in the bodywall muscle through the transparent cuticle. Frames were taken at $10 \mathrm{~s}$ intervals.

DOI: 10.7554/eLife.07366.006 completely disrupted the tubular network after $3 \mathrm{hr}$ (Figure $3 C_{1} D$ ), demonstrating the required catalytic function of VCP. Furthermore, timelapse imaging of Spin-GFP after treatment with $\mathrm{DBeQ}$ for $4 \mathrm{hr}$ revealed that the remaining vesicular lysosome structures are completely static (Video 4).

To determine if the role of VCP in maintaining the tubular lysosomal network in muscle cells is conserved, we overexpressed human VCP in dVCP ${ }^{R N A i}$ muscles. Human VCP should be completely resistant to $d V C P^{R N A i}$ due to lack of extended stretches of nucleotide identity. Overexpressing human $V C P$ in $d V C P^{R N A i}$ muscles rescued the formation of lysosome tubules in every muscle (Figure $3 E-G$ ). These rescue data confirm that VCP knockdown is the cause of disrupted lysosomal network integrity and demonstrate that VCP-dependent activity is conserved in the human VCP protein. 

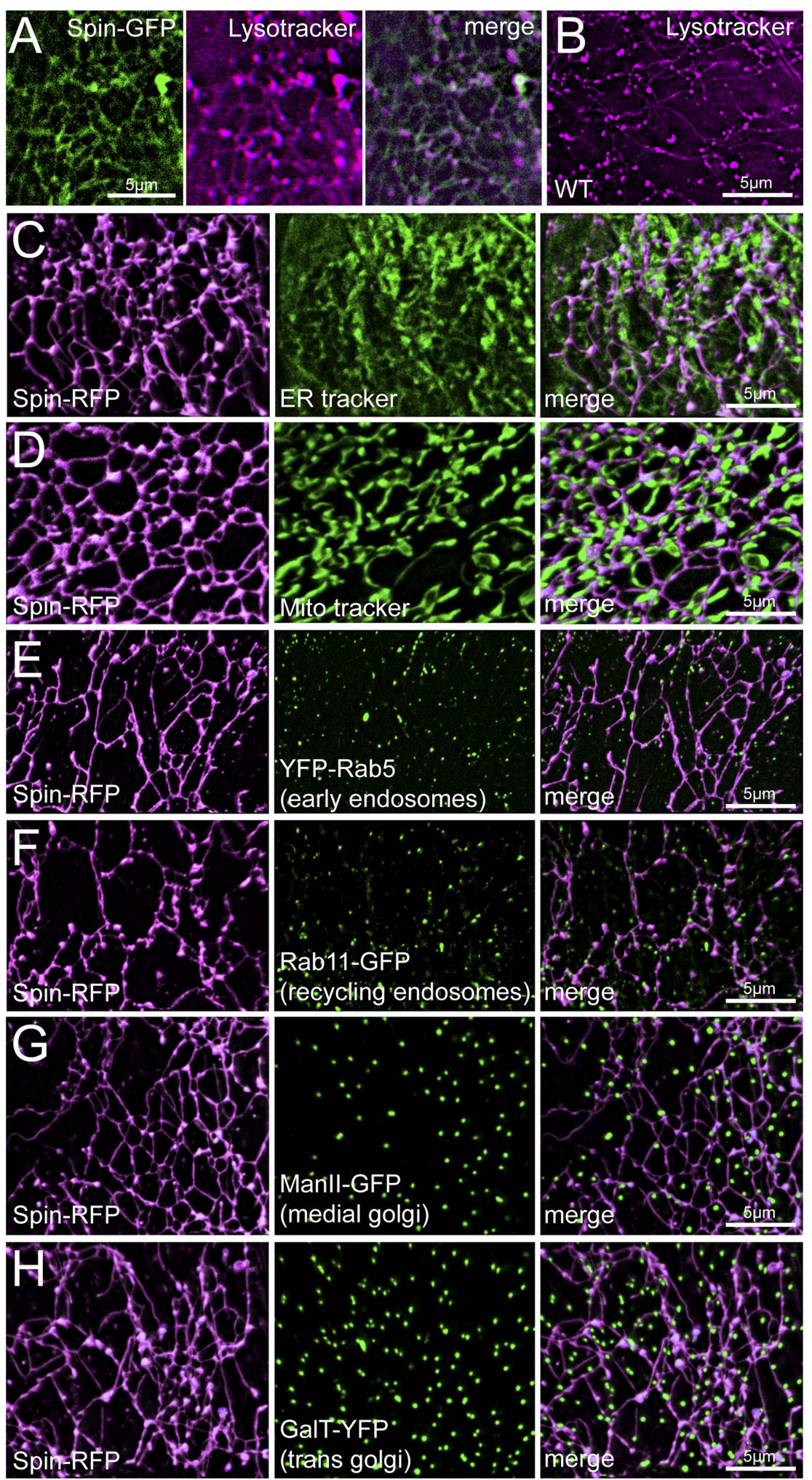

Figure 2. Spin-RFP tubules do not co-localize with mitochondria, ER, golgi or early endosomes. (A) Co-imaging of Spin-GFP and Lysotracker Red staining. (B) Lysotracker staining of wild type muscles. (C-H) Co-imaging of Spin-RFP with ER tracker (C), Mito tracker (D), YFP-Rab5 (E), Rab11-GFP (F), Manll-GFP (G), GalT-YFP (H).

DOI: 10.7554/eLife.07366.007 

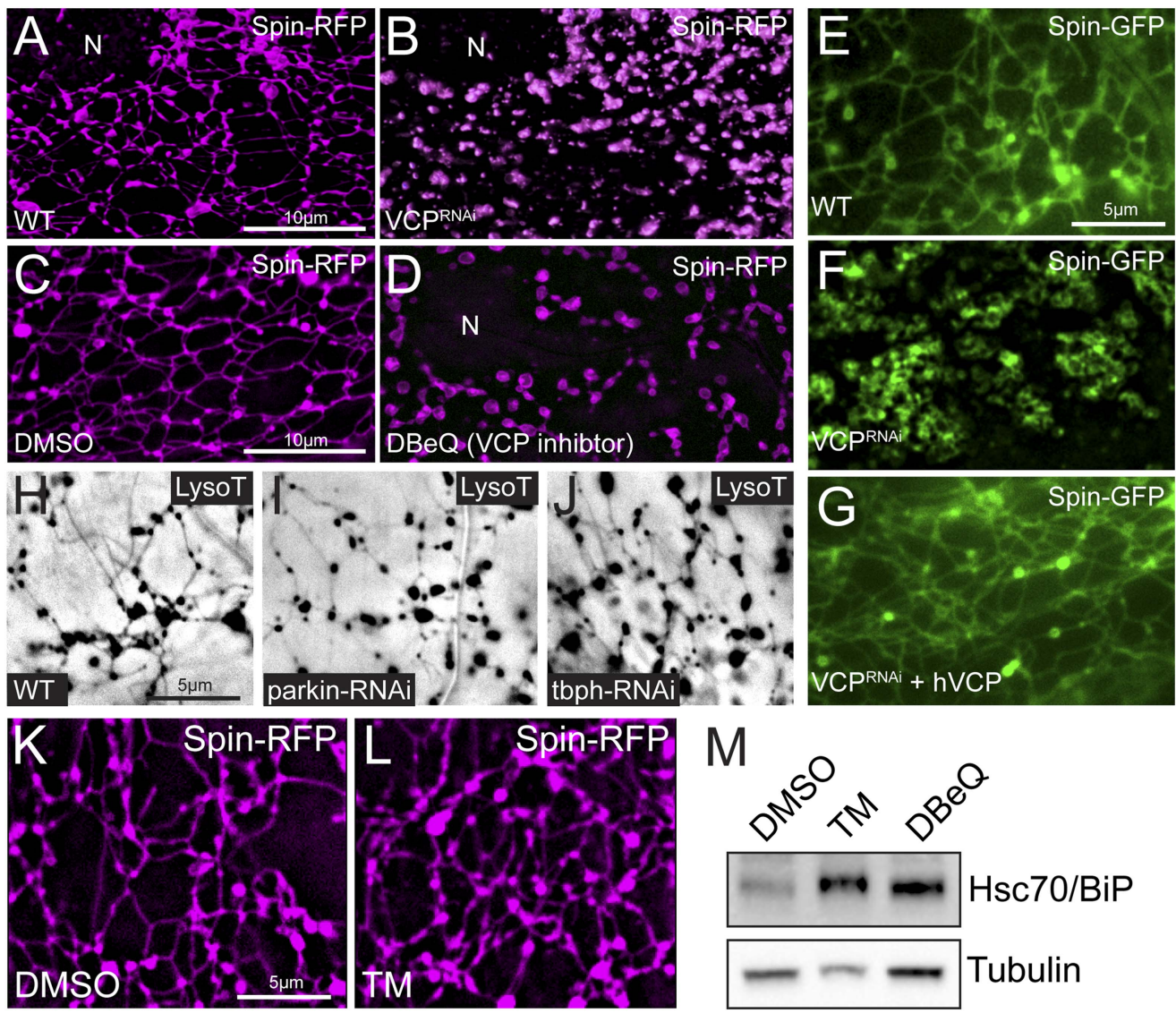

$\mathrm{Hsc70/BiP}$

Figure 3. VCP inhibition disrupts the lysosome tubule lattice and human VCP rescues this defect. (A) Representative live image of Spin-RFP expressed in muscle using the muscle-specific BG57-Gal4 driver. (B) Live image of Spin-RFP in muscles expressing VCP-RNAi using the muscle-specific BG57-Gal4 driver. (C, D) Live images of Spin-RFP expressed in muscles that were treated with DMSO (C) or the VCP-specific inhibitor DBeQ (D) for $4 \mathrm{hr}$. (E) Live image of Spin-GFP expressed in muscles using the muscle-specific BG57-Gal4 driver. (F) Live image of Spin-GFP in muscles expressing VCP-RNAi using the muscle-specific BG57-Gal4 driver. (G) Live image of Spin-GFP in muscles that coexpress VCP-RNAi and human VCP (hVCP) using the muscle-specific BG57-Gal4 driver. (H-J) Lysotracker staining in wild type $(\mathbf{H})$ muscles or muscles expressing parkin-RNAi (I) or tbph-RNAi (J). (K, L) Spin-RFP localization in muscles treated with DMSO (K) or tunicamycin (TM) (L). (M) Western blot analysis of total Hsc70/BiP protein levels. Tubulin serves as a loading control.

DOI: 10.7554/eLife.07366.008

Since VCP knockdown destroys the sarcoplasmic tubular lysosomal network prior to obvious cellular degeneration, we speculated that dismantling of the network could be a precursor to cellular degeneration. However, it is also possible that loss of this network is a secondary correlate of impaired muscle health. To address this issue, we examined two additional RNAi-mediated conditions that cause muscle degeneration, looking for the presence or absence of tubular lysosomes. Specifically, we expressed RNAi against parkin and tbph, Drosophila orthologues of genes linked to Parkinson's and ALS, respectively. These RNAi have been shown to cause muscle degeneration in Drosophila (Diaper et al., 2013; Cornelissen et al., 2014). Remarkably, we did not observe any significant effect on lysosome tubules (Figure $3 H-J$ ) when parkin-RNAi and tbph-RNAi are expressed with BG57-Gal4, the same Gal4 line used to express VCP-RNAi throughout our studies.

VCP has well-established roles in the ERAD pathway and loss of VCP causes ER stress (Wójcik et al., 2006). Thus, it is possible that loss of tubular lysosomes is caused indirectly by ER stress. To address this possibility, we treated muscles with tunicamycin (TM) to induce ER stress by another 


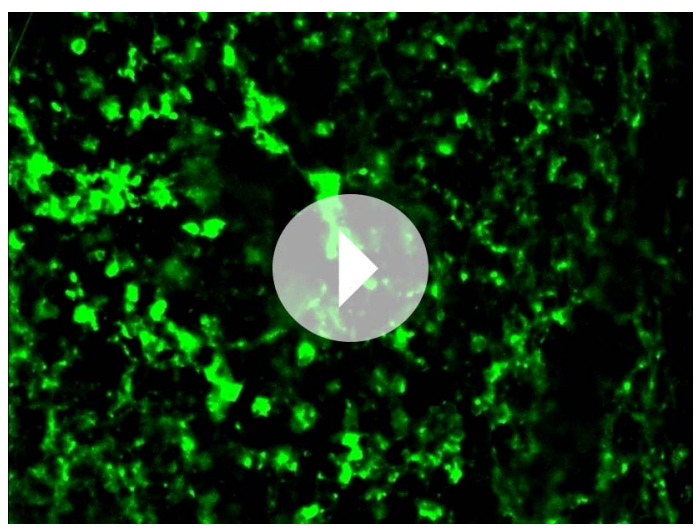

Video 4. Spin-GFP dynamics in muscles expressing VCP-RNAi. Representative time-lapse video of Spin-GFP in muscles expressing VCP-RNAi. Frames were taken at $10 \mathrm{~s}$ intervals.

DOI: 10.7554/eLife.07366.009

\section{Autophagosome membranes co-localize with the tubular lysosome network}

Muscle biopsies from patients with VCP-related disease display an accumulation of cytoplasmic polyubiquitin aggregates (Watts et al., 2004; Weihl et al., 2009; Dolan et al., 2011), suggesting a defect in protein clearance. This led us to explore the intersection of the observed tubular lysosomal network and autophagy. First, we co-imaged Spin-GFP with the autophagosome membrane marker mCherryAtg8a/LC3 to determine the relationship between autophagosomes and the tubular lysosome network. Remarkably, we find that mCherry-Atg8a precisely co-localizes with Spin-GFP-labeled tubules (Figure 4A). The mCherry-Atg8a labeling is widely distributed within the network, but does not label the full extent of every tubule (Figure 4A). Time-lapse imaging revealed mCherry-Atg8a labeling is dynamic within Spin-GFP tubules (Video 5). Some mCherry-Atg8a puncta appear to traffic through the Spin-GFP tubules suggesting that autophagosome membranes and/or cargos are dynamic within the tubular lysosome network (Figure 4B). Atg8 localizes to the autophagophore membrane prior to the formation of an enclosed autophagosome, at which point Atg8 is shed from the outer autophagosome membrane (Xie and Klionsky, 2007). After autophagosomes fuse with lysosomes, lysosomal enzymes degrade the inner autophagosome membrane containing Atg8 (Xie and Klionsky, 2007). Given this sequence of events, we propose that the mCherry-Atg8a positive regions of the lysosome tubules represent ongoing degradation of autophagosome membranes following fusion with the Spin-GFP positive lysosomal network.

Next, we explored the consequence of disrupting VCP activity on Atg8 and Spin-GFP colocalization. When VCP was knocked down and the tubular-lysosomal network was eliminated, Atg8 no longer co-localized with Spin-GFP (Figure 4C). Rather, Atg8-positive vesicles were found closely apposed to Spin-positive, vesicular lysosomal compartments. This close apposition suggests that the autophagosomes can identify and potentially dock against the lysosomal membranes, but fusion of the autophagosome with the lysosome is defective. This is consistent with an established role for the yeast VCP homolog, Cdc48, in membrane fusion (Latterich et al., 1995).

To test whether tubules that are positive for both mCherry-Atg8a and Spin-GFP are, indeed, a consequence of fused auto-lysosomes, we imaged a dual fluorescent reporter GFP-mCherry-Atg8a (Nezis et al., 2010). In neutral pH conditions, both GFP and mCherry fluoresce. However, in acidic environments such as in the lysosomal lumen, GFP fluorescence is quenched and only mCherry is observed. Thus, autophagosomes that are fused with lysosomes will exhibit mCherry but not GFP fluorescence. It is important to note that Spin-GFP fluorescence is not quenched because the C-terminal GFP tag resides on the cytoplasmic face of lysosomes (Dermaut et al., 2005). When we expressed the dual fluorescence reporter in muscles we found no detectable GFP fluorescence in the tubules that label strongly for mCherry-Atg8a (Figure 4D), consistent with other data indicating that 

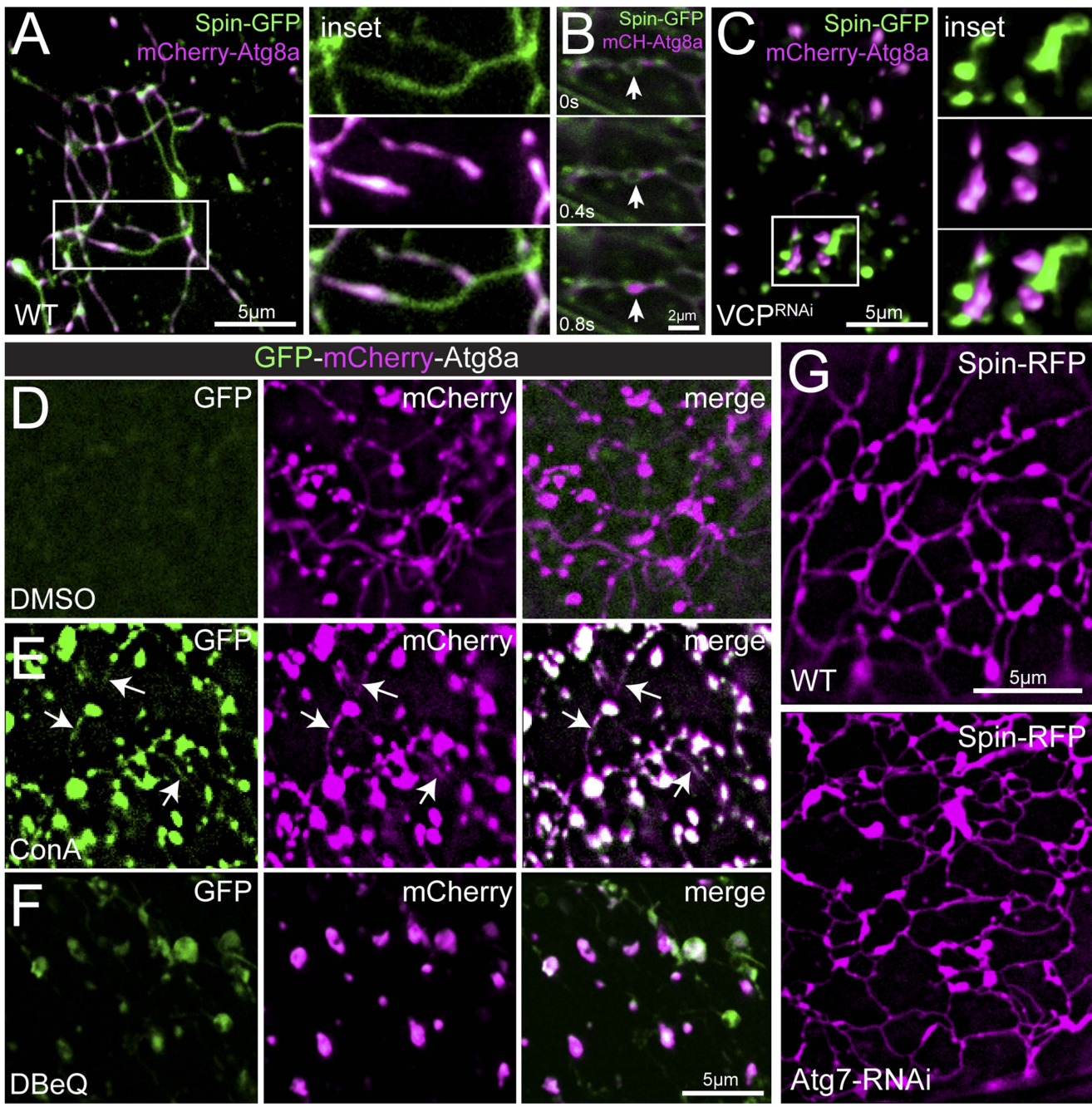

GFP-mCherry-Atg8a
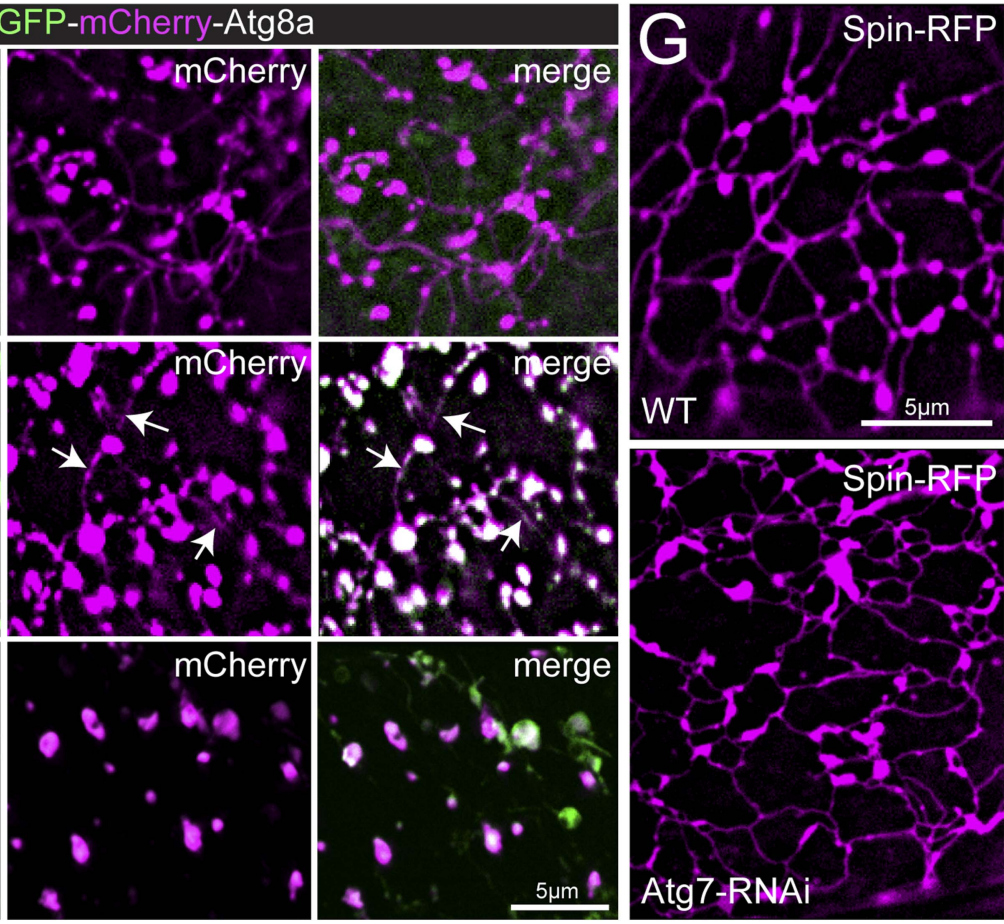

Figure 4. Autophagosomes co-localize with the tubular lysosomal network. (A) Representative live image of SpinGFP and mCherry-Atg8a co-expressed in muscles using the muscle-specific BG57-Gal4 driver. (B) Representative time-lapse sequence of Spin-GFP and mCherry-Atg8a in muscle. The arrow follows a mCherry-Atg8a positive puncta trafficking along a Spin-GFP tubule. (C) Spin-GFP and mCherry-Atg8a no longer co-localize in muscles expressing VCP-RNAi. White box indicates region shown at higher magnification and separate channels at right. $\mathbf{D}$. Live image of GFP-mCherry-Atg8a in muscles treated with DMSO for $3 \mathrm{hr}$. Note the lack of GFP signal. (E) Live image of GFP$m$ Cherry-Atg8a in muscles treated with the V-ATPase specific inhibitor Concanamycin A (ConA) for 3 hr. Note the presence of GFP-positive tubules. (F) Live image of GFP-mCherry-Atg8a in muscles treated with the VCP-specific inhibitor $\mathrm{DBeQ}$ for $3 \mathrm{hr}$. Note the presence of GFP-positive vesicles. (G) Spin-RFP localization in WT muscles or muscles expressing Atg7-RNAi using the muscle specific BG57-Gal4 driver.

DOI: 10.7554/eLife.07366.010

the tubules are acidic. To further test the acidic nature of the tubules, we treated muscles with concanamycin A, a specific inhibitor of the lysosomal V-ATPase that is required for lysosome acidification (Huss et al., 2002). Upon treatment with ConA for $3 \mathrm{hr}$, we observed GFP and mCherry positive tubules (Figure 4E). Together, these data further verify that the lysosome tubules are acidic and also demonstrate that acidification is not necessary to maintain the structure of the tubules.

Again, we explored the consequence of disrupting VCP activity. Inhibiting VCP function with DBeO created GFP-positive vesicles (Figure 4F) that must be non-acidified compartments, a finding that is consistent with the existence of autophagosomes that have not yet fused with Spin-positive lysosomes. Since the appearance of GFP-positive vesicles occurs in a time frame of minutes to hours, it 


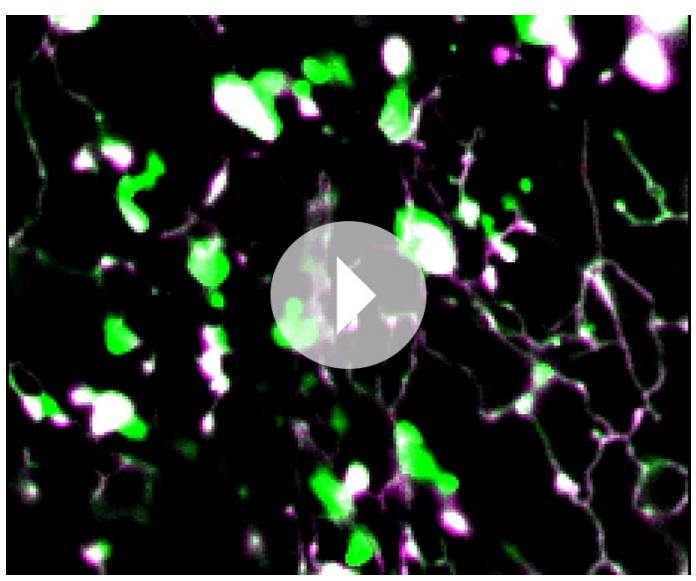

Video 5. Spin-GFP and mCherry-Atg8a dynamics in Drosophila muscle. Representative time-lapse video of Spin-GFP and mCherry-Atg8 co-expressed in muscles. Frames were taken at $10 \mathrm{~s}$ intervals. DOI: 10.7554/eLife.07366.011

localizes diffusely at the nucleus and throughout the cytoplasm (Vesa et al., 2009; Tresse et al., 2010a; Wang et al., 2011). To examine VCP localization in live muscles, we generated a UAS-VCP-Venus transgenic fly. Similar to previous reports for mammalian VCP, we observed abundant VCP localization in and around the nucleus and diffusely in the cytoplasm (Figure 5-figure supplement 1). But, VCP-Venus also concentrated at structures that are labeled by either Spin-RFP (Figure 5A) or mCherry-Atg8a (Figure 5B), demonstrating that VCP localizes to auto-lysosomes.

Because inhibiting the catalytic function of VCP leads to tubule deterioration, we tested whether inhibiting the catalytic function of VCP would affect its localization to auto-lysosomes. Surprisingly, inhibiting VCP activity with $\mathrm{DBeQ}$ for $2 \mathrm{hr}$ triggered the formation of rod-shaped VCP aggregates that effectively sequester VCP-Venus from the sarcoplasm (Figure 5C). The formation of these aggregates is striking, as they resemble rod-like structures characteristic of prion aggregates. We considered two possible reasons that application of $\mathrm{DBeO}$ might increase the propensity for VCP to aggregate. First, $\mathrm{DBeO}$ binding to VCP might initiate aggregation directly by altering the solubility of VCP. Alternatively, the aggregation could be caused by the functions of VCP in other contexts, such as proteasome-dependent protein degradation. Remarkably, when we applied the proteasome inhibitor MG132, VCP-Venus rapidly aggregated in a manner identical to that observed following $\mathrm{DBeQ}$ incubation (Figure 5D). VCP aggregation was not due to increased VCP protein levels as a result of proteasome inhibition, because total VCP protein did not increase significantly upon MG132 or DBeQ treatment (Figure 5E).

While the significance of VCP-Venus aggregates remains uncertain, this phenotype provided us with a means to rapidly and reversibly sequester VCP protein and control its access to auto-lysosomal membranes. Since VCP exists as a hexamer, MG132 incubation in muscles over-expressing VCP-Venus should sequester both wild type and Venus-tagged VCP. Application of MG132 induced VCP-Venus aggregate formation, which correlated with dissolution of the tubular lysosomal network (Figure 5D). When MG132 was washed out, VCP aggregates dissolved within $20 \mathrm{~min}$ and, as they disappeared, cytoplasmic VCP fluorescence intensity increased (Figure 5F and Video 6). During this time, cytoplasmic VCP-Venus accumulated at autophagosomes/lysosomes (mCherry-Atg8a) and tubules began to reform (Figure 5F and Video 6). These data indicate that VCP localizes to auto-lysosomes, where it could participate in auto-lysosomes tubulation.

\section{Disrupted lysosome tubules correlate with muscle weakness and autophagy/lysosome defects}

We next investigated the consequence of disrupting the auto-lysosome tubule network. We first examined overall muscle function. When VCP RNAi was expressed specifically in muscle, muscle 

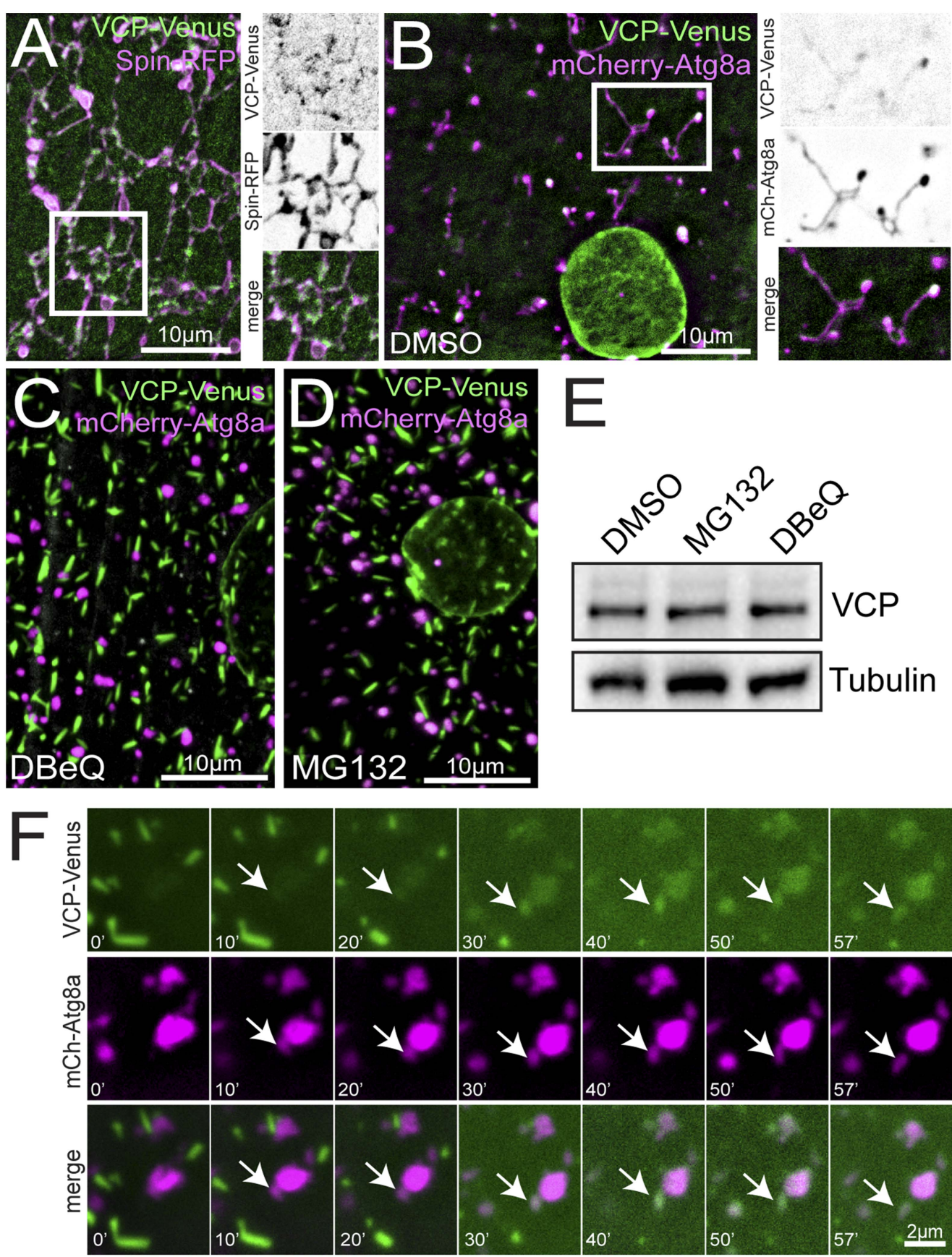

Figure 5. VCP co-localizes with the tubular auto-lysosomes. (A) Representative live image of VCP-Venus and SpinRFP expressed in muscles using the muscle-specific BG57-Gal4 driver. White box indicates region shown at higher magnification and separate channels at right. (B) Representative live image of VCP-Venus and mCherry-Atg8a expressed in muscles using the muscle-specific BG57-Gal4 driver. Inset as in A. (C, D) VCP-Venus and mCherryAtg8a localization in muscles treated with the VCP inhibitor DBeQ (C) or the proteasome inhibitor MG132 (D) for 3 hr. (E) Western blot analysis of total VCP protein levels from muscles in the treatments indicated. Tubulin serves as a loading control. (F) Representative time-lapse sequence of VCP-Venus and mCherry-Atg8a after MG132 was washed out. The arrow indicates a tubule extending from a mCherry-Atg8a positive vesicle. DOI: 10.7554/eLife.07366.012

Figure 5. continued on next page 
Figure 5. Continued

The following figure supplement is available for figure 5 :

Figure supplement 1. VCP-Venus localization in Drosophila muscle.

DOI: 10.7554/eLife.07366.013

wasting was apparent and third instar larvae exhibited a severe impairment in their ability to move (Figure 6-figure supplement 1A,B). When prodded, the animals would move, but their movements were slow and only lasted for short periods of time. The defect in their motility is not due to defects in the nervous system because synaptic transmission at the NMJ remained intact (Figure 6-figure supplement $1 C, D)$. These data are consistent with compromised muscle function that parallels the muscle weakness observed in human VCP-related diseases.

Next, we investigated the degradation capacity of the collapsed tubular lysosomes. For lysosomes to degrade their cargo they must be acidified and the proteolytic enzymes must be present. We first examined the acidification of the lysosomes by co-imaging Spin-GFP with Lysotracker in VCP-RNAi animals and found that the enlarged Spin-GFP vesicles also co-stained with Lysotracker (Figure 6-figure supplement 2A), indicating that they are acidic. Then, we examined whether lysosome enzymes were delivered properly to the lysosomes. Normally, the lysosomal enzyme Cathepsin- $L$ is proteolytically processed in the lysosomal lumen to form a mature enzyme. To further examine the functionality of the lysosomes, we examined processing of Cathepsin- $L$ and found no significant difference in Cathepsin-L processing in VCP-RNAi animals or animals treated with DBeQ (Figure 6-figure supplement 1B). Thus, disruption of the tubular lysosomal network does not appear to affect the proteolytic capacity of sarcoplasmic lysosomes.

To this point, our data suggest that loss of VCP disrupts the fusion of autophagosomes with functional lysosomes and, in parallel, causes the collapse of the tubular lysosomal network. Based on this, we expected to find evidence of failed autophagy in VCP knockdown muscle. In wild type muscles stained with a poly-ubiquitin antibody, we observed small puncta around the nucleus and a few small puncta in the cytoplasm (Figure 6A). These small puncta likely represent active sites of protein degradation by the proteasome. However, in muscles expressing VCP-RNAi we observed a dramatic accumulation of cytoplasmic poly-ubiquitin aggregates (Figure 6B). Even acute treatment with $\mathrm{DBeQ}$ was sufficient to produce cytoplasmic poly-ubiquitin aggregates (Figure 6C-E). We also note that VCP inhibition caused a dramatic decrease in poly-ubiquitin conjugates around the

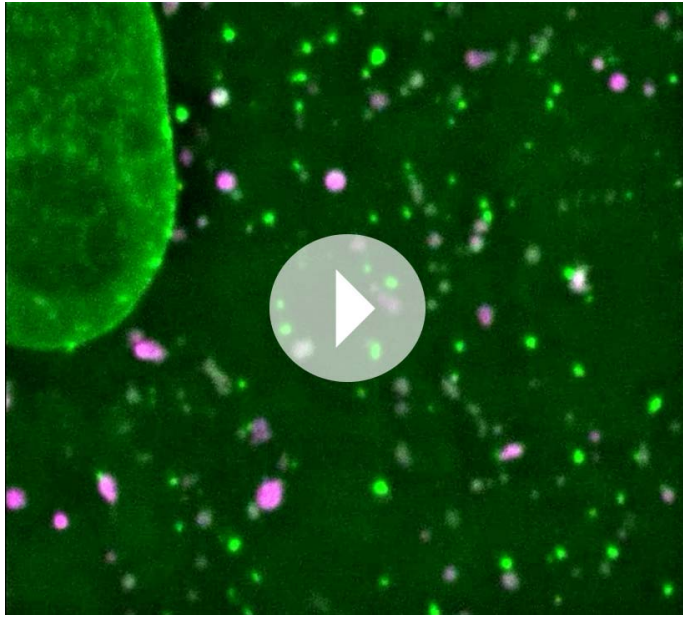

Video 6. VCP-Venus and mCherry-Atg8a dynamics after MG132 wash out. Muscles co-expressing VCPVenus and mCherry-Atg8 were treated with the proteasome inhibitor MG132 for $3 \mathrm{hr}$. MG132 was washed out and time-lapse images were taken every $10 \mathrm{~s}$ DOI: 10.7554/eLife.07366.014 nucleus, which likely reflects failed delivery of poly-ubiquitinated proteins to the proteasome. Importantly, we find that Spin-positive lysosomes are devoid of poly-ubiquitin staining (Figure 6F), an observation that is consistent with our model of failed fusion of autophagosomes with lysosomes.

In addition to clearing protein aggregates from the cytoplasm, autophagy is also responsible for clearing damaged organelles, including mitochondria. We examined the functional pool of muscle mitochondria in vivo using mitotrackerOrange CM-H2TMRos, which selectively stains mitochondria with an active membrane potential. Muscles expressing VCP-RNAi displayed less mitotracker-Orange CM-H2TMRos staining and the visualized mitochondria had an altered morphology, appearing round and dispersed rather than being densely packed, tubular structures (Figure 6G,H). The swollen mitochondria observed in the VCP RNAi expressing muscles 

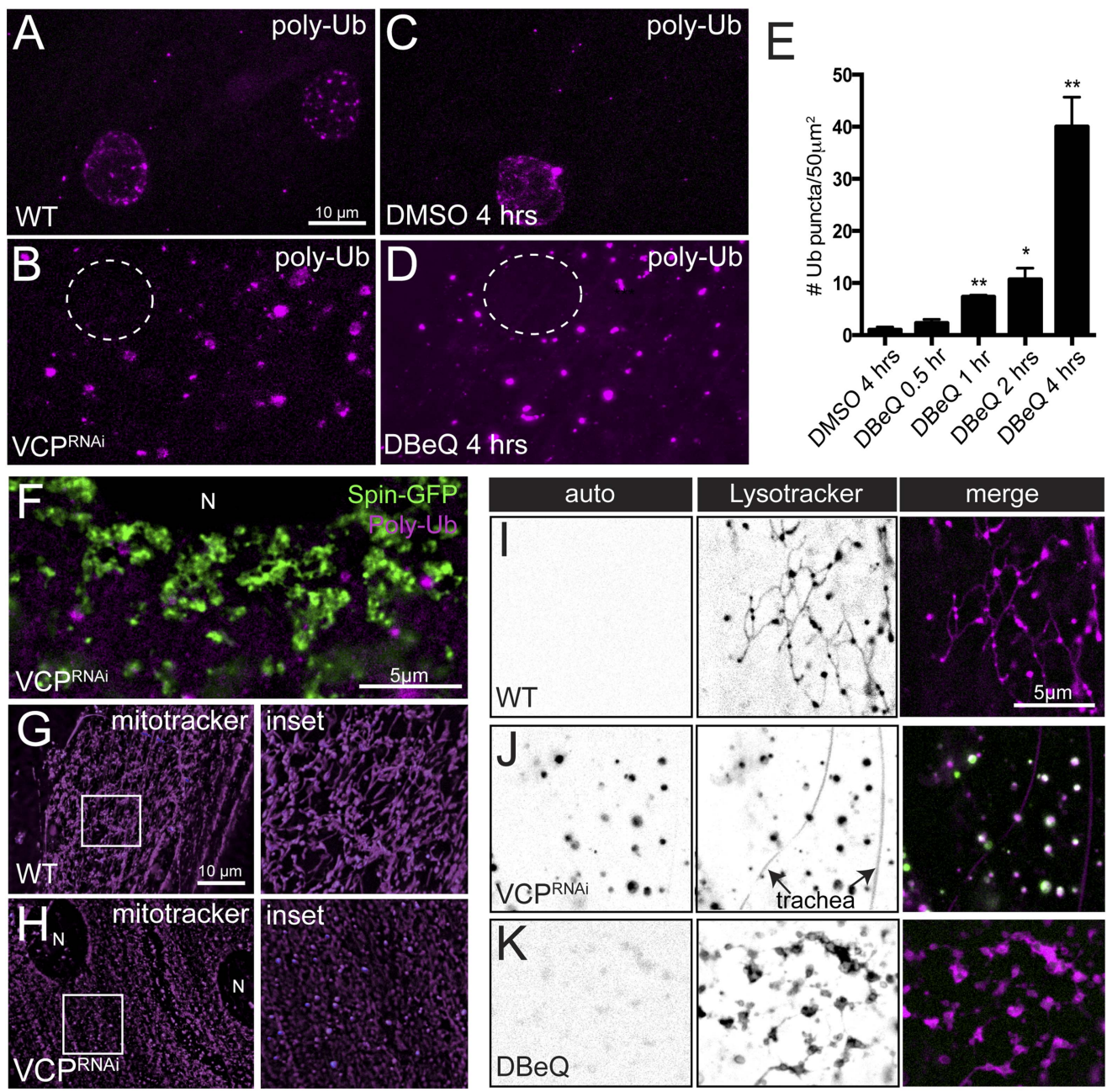

Figure 6. Disruption of the tubular auto-lysosomal network correlates with increased poly-Ubiquitin aggregates, impaired mitochondria and increased lipofuscin granules. (A, B) Wild type (A) and VCP-RNAi (B) expressing muscles were fixed and stained with a poly-Ubiquitin antibody. Nuclei with localized poly-Ubiquitin staining are apparent in A. Nuclei are indicated (dashed circle) in B. (C, D) Wild type animals were treated with DMSO (C) or the VCP-specific inhibitor $\mathrm{DBeQ}(\mathbf{D})$, fixed and stained with a poly-Ubiquitin antibody. (E) Quantitation of the number of polyUbiquitin aggregates per $50 \mu \mathrm{m}^{2}$ from wild type muscles treated with DMSO for $4 \mathrm{hr}$ or DBeQ for various times ( $n=9,{ }^{*} p<0.05,{ }^{* \star} p<0.01$ ). (F) Localization of Spin-GFP and poly-Ubiquitin in muscles expressing VCP-RNAi. (G, H) Mitotracker-C2TMRos staining in control $(\mathbf{G})$ and VCP-RNAi $(\mathbf{H})$ muscles. (I-K) Autofluoresence at $488 \mathrm{~nm}$ and lysotracker staining in wild type (I), muscles expressing VCP-RNAi $(\mathbf{J})$, and wild type muscles treated with the VCPspecific inhibitor $\mathrm{DBeQ}$ for $4 \mathrm{hr}(\mathrm{K})$.

DOI: 10.7554/eLife.07366.015

The following figure supplements are available for figure 6:

Figure supplement 1. Loss of tubular lysosomes correlates with impaired muscle function. DOI: 10.7554/eLife.07366.016

Figure supplement 2. Lysosomal acidity and Cathepsin processing are maintained in VCP-RNAi expressing muscles.

DOI: 10.7554/eLife.07366.017

are likely defective and should be a prime target for mitophagy-dependent degradation. Taken together with the appearance of polyubiquitin aggregates, these data are consistent with an overall defect in autophagic clearance of proteins and defective organelles. However, since VCP has also 
been shown to be recruited directly to damaged mitochondria (Kim et al., 2013) we cannot rule out the possibility that the effects on mitochondrial morphology and membrane potential are due to direct VCP functions at the mitochondrial outer membrane.

Finally, we noted an accumulation of lipofuscin granules in VCP-RNAi expressing muscles. Lipofuscin granules are a conglomerate of polymerized non-degradable proteins and lipids that build up in the lysosomal lumen (Szweda et al., 2003). A distinguishing property of lipofuscin granules is that they exhibit auto-fluorescence at $488 \mathrm{~nm}$. Wild type or VCP-RNAi muscles were stained with LysoTracker-Red and imaged at both $488 \mathrm{~nm}$ (green) and $555 \mathrm{~nm}$ (magenta). The wild type muscles did not display any detectable auto-fluorescence at $488 \mathrm{~nm}$ (Figure 6I). But, we observed strong autofluorescent puncta in VCP-RNAi that co-localized with LysoTracker (Figure $6 \mathrm{~J}$ ), indicative of lipofuscin granules. We did not observe autofluorescent puncta to the same extent when wild type muscles were treated with $\mathrm{DBeO}$ for $4 \mathrm{hr}$ (Figure $6 \mathrm{~K}$ ), suggesting that lipofuscin accumulation is a progressive phenotype. Alternatively, lipofuscin granule accumulation could require loss of VCP protein rather than just loss of VCP catalytic activity. These data suggest that maintaining the structural integrity of lysosome tubules is critical for lysosome function.

\section{VCP-related disease mutations disrupt lysosome tubules}

Finally, we asked whether overexpression of VCP transgenes that harbor disease-causing mutations impairs the presence or dynamics of tubular lysosomes. To date, a total of 19 missense mutations in 13 different residues are associated with IBMFD that reside in either the Cdc48 homology domain, the L1 linker domain or the D1 ATPase domain (Figure 7A and [Ju and Weihl, 2010]). We selected one mutation from each of these three domains to examine the effect on lysosome tubules: R155H, R1910 and A232E. R155 is the most common hereditary mutation and is located in the CDC48 homology domain, which is a protein interaction module that plays an important role in VCP substrate binding (Ju and Weihl, 2010). R191 is located in the linker region between the CDC48 domain and the first ATPase catalytic domain (D1). A232 is located at the beginning the of the D1 domain and is the most severe clinical mutation (Watts et al., 2004). All three residues are conserved in the Drosophila protein (Figure 7A). As a control, we show that over-expression of wild type dVCP does not alter tubular lysosomes (Figure 7B). We then demonstrate that over-expression of each of the mutant VCP transgenes profoundly impairs the tubular lysosomal network (Figure 7C-F). In parallel, we find an increase in sarcoplasmic auto-fluorescence when these VCP mutants are over-expressed (Figure 7B-G). Overexpression of the A229E mutation, which is the most severe clinical mutation, caused the largest increase in auto-fluorescence compared to the other clinical mutations (Figure 7F). Because over-expression of the disease relevant VCP transgenes phenocopies VCP-RNAi expression, these data suggest that the disease mutations are dominant interfering mutations. Thus, overexpression of VCP disease-associated mutations disrupts lysosome tubules in vivo, an effect that causes accumulation of cytoplasmic lipofuscin granules.

\section{Discussion}

Here we demonstrate that lysosomes form a dynamic, tubular array that extends throughout the sarcoplasm of Drosophila muscle, in vivo. To our knowledge, this is the first observation of such an extensive, dynamic tubular lysosomal network in any in vivo system. We define this as a lysosomal network because it has a low $\mathrm{pH}$, it is labeled by the late endosomal marker Spin-GFP, does not colocalize with the ER, golgi apparatus, early endosomes or recycling endosomes, and we find that mCherry-Atg8 traffics to this compartment, indicative of autophagosome fusion with a network of tubular lysosomes. Lysosomal network dynamics require an intact microtubule cytoskeleton, clathrin heavy chain, and VCP. We provide evidence that the catalytic activity of VCP is continuously required to sustain network integrity. When VCP is depleted or inhibited, the lysosomal network collapses and both cellular proteostasis and autophagy are compromised. Collapse of the network correlates with cellular manifestations of VCP-associated degenerative diseases, including the appearance of protein inclusion bodies (Kimonis et al., 2008; Weihl et al., 2009). While many questions remain unanswered, our discovery of tubular lysosome dysfunction following overexpression of pathogenic VCP mutants represents one the earliest markers of VCP-dependent muscle pathology. Further characterization could pave the way toward methods that might beneficially stabilize lysosomal function and ameliorate the progression of widespread degenerative disease. 


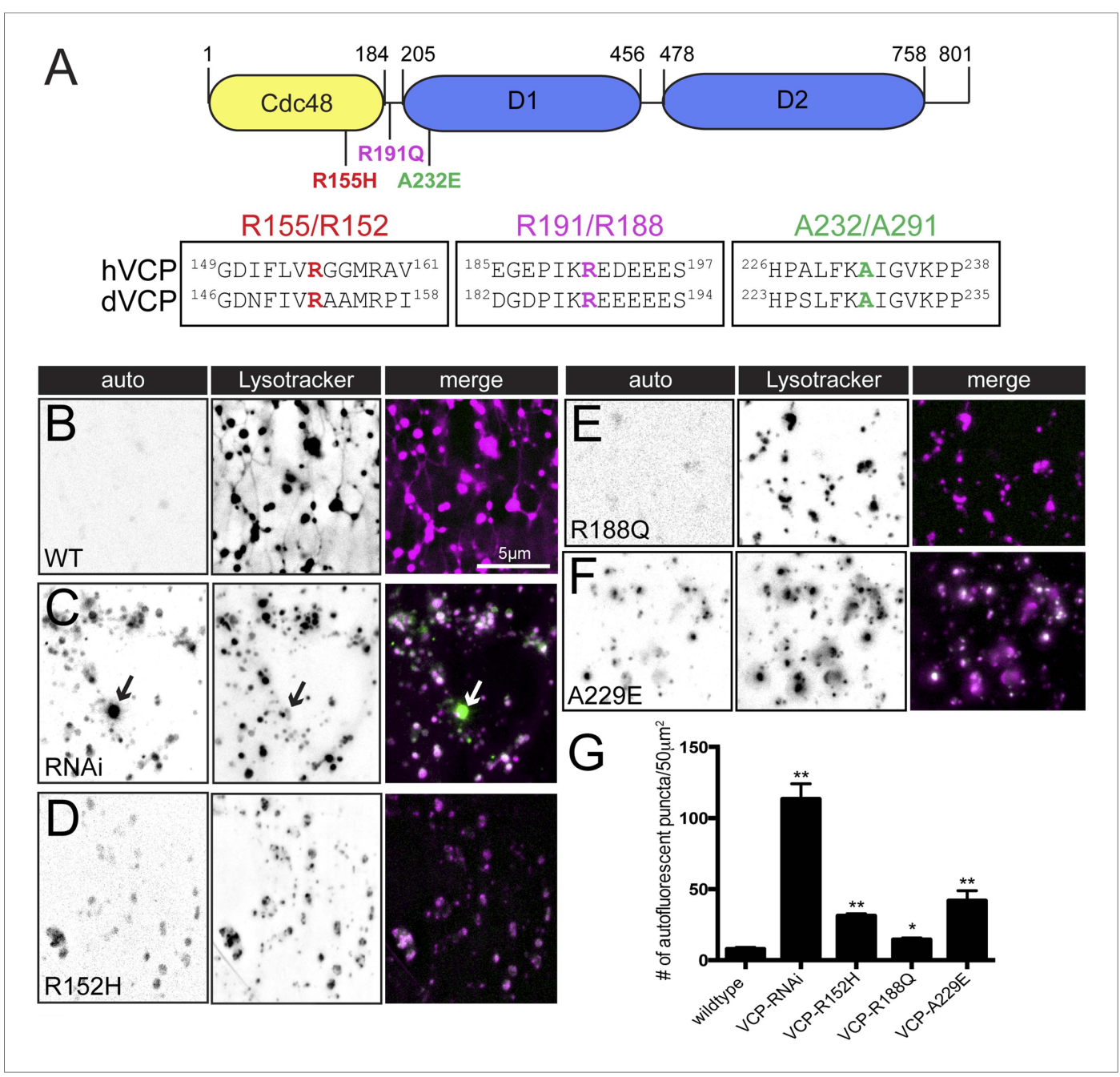

Figure 7. Pathogenic VCP alleles disrupt the tubular auto-lysosomal network. (A) Schematic diagram of VCP protein. Top: Human pathogenic VCP mutations are labeled on the cartoon. Bottom: sequence alignment of human VCP and Drosophila VCP/Ter94 pathogenic mutant regions. (B-F) Autofluoresence at $488 \mathrm{~nm}$ and lysotracker staining in wild type muscles expressing VCP-WT (B), VCP-RNAi (C) VCP-R152H (D), VCP-R188Q (E) or VCPA-229E (F) transgenes. (G) Quantitation of the number of auto-fluorescent puncta per $50 \mu \mathrm{m}^{2}$ in the genotypes indicated $\left(n=9,{ }^{*} p<0.05\right.$, $\left.{ }^{* *} p<0.01\right)$.

DOI: 10.7554/eLife.07366.018

\section{Role of an extended tubular lysosome network in muscles}

In most tissues, autophagy is induced upon nutrient starvation, but muscles are one of the unique tissues in which autophagy occurs in the absence of starvation (Mizushima et al., 2004). In fact, basal autophagy is required to maintain muscle mass (Masiero et al., 2009). The constitutive levels of autophagy in muscle are likely related to the large energy requirement of muscles compared to other cell types and their ability to serve as a source of metabolic energy for other organs (Sandri, 2010). Muscle sarcoplasms are also unique in that they are shared between multiple nuclei and are much larger in volume compared to many other cell types. We propose that the observed extended, lysosomal network that is maintained by VCP is a cellular solution to ensure highly efficient autophagy throughout the entirety of muscle sarcoplasms. It is as if the lysosomes vascularize the sarcoplasm, ensuring that no portion of the sarcoplasm is ever far from a lysosomal depot where autophagic cargo can be degraded. Likewise, this network could facilitate the local recycling of nutrients throughout the sarcoplasm to effectively meet local demands. A similar argument can be made regarding the autophagy-dependent turnover of mitochondria, which are densely distributed throughout the 
muscle. In support of this, VCP is critical for the rapid degradation of muscle proteins during muscle atrophy and expression of a dominant negative form of VCP reduces protein degradation by both proteasomes and lysosomes (Piccirillo and Goldberg, 2012).

Lysosomal membranes have been observed to extend tubules and fission off to produce de novo lysosomes following fusion of autophagosomes with lysosomes (Yu et al., 2010). This process, termed ALR, serves as a mechanism to recycle lysosomes when autophagic demand is high (Yu et al., 2010). It is unknown whether ALR is a constitutive process that participates in ongoing, steady-state proteostasis in vivo or whether it is a process that is specifically induced following stress-induced autophagy. It is possible that the extended, dynamic lysosomal tubule network that we observe is related to ALR, perhaps acting as a platform for efficient ALR throughout the sarcoplasm. In support of this, the mammalian Spinster homolog is required for ALR (Rong et al., 2011). However, the relatively low frequency with which we observed scission events and the formation of new small lysosome vesicles is not entirely consistent with this idea.

The tubular network identified in this study more closely resembles the lysosome tubular networks that have been observed in cultured macrophage cells (Swanson et al., 1987a, 1987b; Knapp and Swanson, 1990). Macrophage lysosome tubules can form a web of tubules that appear to be stably connected throughout the cytoplasm (Swanson et al., 1987b). The purpose of lysosome tubules in this system is still mysterious. We have now identified specific defects associated with loss of lysosome tubules and our data suggest that the tubular network may distribute auto-lysosomal activity throughout the cell. Clues to the function of tubular lysosomes might also be gleaned from studies of early endosomes. Early endosomes form vesicular-tubular structures similar to what we have observed here (Huotari and Helenius, 2011). In early endosomes it has been established that tubules can be discrete membrane compartments with a different lipid composition and cargo compared to that within the vesicular body of the early endosome (Huotari and Helenius, 2011). By analogy, the ability of lysosomes to exist in a tubular-vesicular state in cellular contexts where their functional demand is high, might allow lysosomes to execute diverse functions more efficiently through compartmentalization.

\section{Consequences of tubular lysosome dysfunction}

VCP and the yeast homologue Cdc48 have been ascribed many functions within the cell including cell cycle progression (Moir et al., 1982), UPS and ERAD protein degradation (Meyer et al., 2012; Wolf and Stolz, 2012), mitophagy (Taylor and Rutter, 2011) and classical autophagy (Ju et al., 2009; Tresse et al., 2010a; Dargemont and Ossareh-Nazari, 2012; Meyer et al., 2012). VCP achieves these diverse activities, in part, through its generalized function as an ubiquitin-dependent 'segregase' that dissociates protein conjugates tagged with ubiquitin from protein complexes and organelle membranes (Halawani and Latterich, 2006; Meyer et al., 2012). For each process that relies upon VCP activity, different cofactors control VCP localization and function (Baek et al., 2013). To this list of VCP-mediated activities, we now add the action of VCP in controlling the integrity and dynamics of a tubular lysosomal system and fusion of autophagosomes with tubular lysosomes. Additional, future experimentation will be necessary to determine which specific VCP-mediated molecular mechanism(s) is most directly relevant to the integrity and dynamics of lysosomal tubules in muscle.

The phenotypic consequences following the loss or inhibition of VCP in Drosophila muscle include the collapse of the tubular lysosomal network, failed fusion of autophagosomes with lysosomes, accumulation of sarcoplasmic poly-ubiquitin aggregates, accumulation of lipofuscin granules, impaired mitochondria and impaired muscle health. All of these phenotypes are hallmarks of degenerative diseases that are associated with mutations in VCP (Watts et al., 2004). We can now ascribe some of these phenotypes to the action of VCP at lysosomal membranes. Our data indicate that VCP localizes to autolysosomes and loss of VCP causes collapse of the tubular lysosomal network and failed autophagosome-lysosome fusion. Furthermore, when previously sequestered VCP is released back into the cytoplasm, VCP translocates to dormant autolysosomes and tubulation ensues. Although we cannot distinguish the role of VCP in the initiation and/or maintenance of lysosome tubules, these data argue that VCP acts at the autolysosmal membrane to control autolysosomal dynamics and the progression of autophagic protein clearance. If VCP is necessary for normal activity of the autophagy-lysosome system in muscle as our data suggests, then it seems likely that the accumulation of poly-ubiquitin aggregates and lipofuscin granules are a direct consequence of 
impaired VCP-dependent lysosomal function. This assertion is further supported by our demonstration that over-expression of pathogenic VCP mutant transgenes disrupt the tubular lysosomal network and also cause accumulation of ubiquitin and lipofuscin material. Importantly, these transgenes in Drosophila do not appear to disrupt VCP roles in the UPS or ERAD pathways, emphasizing the role of impaired autophagy in pathogenic VCP phenotypes (Tresse et al., 2010b; Chang et al., 2011). We acknowledge that lipofuscin granule accumulation following overexpression of pathogenic VCP mutant transgenes could represent a byproduct of proteotoxic stress caused by defects in the UPS or autophagy/lysosome pathways that eventually lead to the transformation of proteins into nondegradable products. Ultimately, more detailed biochemical analyses will be required to elucidate precisely how VCP functions at lysosome membranes and how this activity might be coordinated with other aspects of VCP function throughout the cell. Taken together, our identification of tubular lysosomes in Drosophila muscle challenges the traditional view of vesicular lysosomes and suggests that lysosome structures can be more versatile than previously assumed. Understanding how lysosomes regulate their morphological state will be an exciting avenue for future studies.

\section{Materials and methods}

\section{Experimental procedures}

Fly stocks

The following transgenic fly stocks were generated in this study: UAS-Spin-RFP, UAS-Spin-GFP and UAS-Ter94/VCP-Venus. Drosophila spinster and ter94 cDNA were obtained by amplifying from DGRC (Drosophlia Genomics Resource Center, Bloomington, IN) clones AT25382 and GM02885 respectively. The cDNAs were cloned into the Gateway pENTR vector (Invitrogen, South San Francisco, CA) and subsequently cloned into destination vector pTWV obtained from the Drosophila Gateway Vector Collection (Carnegie Institution, Baltimore, MD). Transgenic animals were then generated by BestGene. UAS-mCherry-Atg8a and UAS-GFP-mCherry-Atg8a stocks were purchased from the Bloomington Stock Center. UAS-RNAi stocks were purchased from the Vienna Drosophila Resource Center. Pathogeneic UAS-Ter94 alleles were a kind gift from TK Sang (National Tsing Hua University, Hsinchu, Taiwan). Golgi markers UAS-Manll-GFP and UAS-GalT-YFP were a kind gift from B Ye (University of Michigan, Ann Arbor, MI). Gal4 muscle drivers used in this study include: BG57-Gal4 and MHC-Gal4.

\section{Microscopy methods}

Third instar larvae were dissected in Schneider's insect cell media (Gibco, Grand Island, NY) supplemented with 10\% FBS (Gibco) and Penicillin/Streptomycin (Gibco) and all live imaging was performed in insect cell media. For all imaging experiment, at least 3 muscles in 3 animals were imaged to account for variances between muscles and animals and the most representative images are shown in each figure $(n \geq 9)$. The following drugs were diluted in insect cell media to the following final concentrations: 1 uM LysoTracker Red DND-99 (Life Technologies, South San Francisco, CA), 1uM ER Tracker green (Life Technologies), 10uM Nocodazole (Sigma, St. Louis, MO), 10uM Latrunculin A (Life Technologies), 10uM DBeQ (Sigma), 1uM MG132 (Sigma), 100 nM Concanamycin A (Santa Cruz Biotechnology, Dallas, TX), 2ug/ml TM (Sigma) and $500 \mathrm{nM}$ Mitotracker-Orange-CM-H2TMRos (Life Technologies). LysoTracker, ER Tracker and Mitotracker-Orange-CM-H2TMRos were incubated on the dissected larvae for $1 \mathrm{hr}$ prior to imaging. Nocodazole, LatA, DBeQ, TM, Concanamycin A and MG132 were incubated on the dissected larvae for 3-4 hr prior to imaging.

For Ubiquitin staining, larvae were dissected and fixed with 4\% PFA for $15 \mathrm{~min}$. After fixation, larvae were washed $4 \times$ with PBS-T and incubated with anti-poly-Ubiquitin (Thermo Scientific, Waltham, MA) at 1:1000 dilution overnight at $4^{\circ} \mathrm{C}$. Larvae were washed again $4 \times$ with PBS-T, incubated with a fluorescently labeled secondary antibody at 1:5000 dilution (Life Technologies), and washed again $4 \times$ with PBS-T before mounting on a slide with vectashield for imaging.

Imaging was performed on an inverted Axiovert 200 microscope (Zeiss) using a 100x Plan Apochromat objective (1.4NA). Images were captured with a CoolSnap HQ2 CCD camera (Photometrics) and de-convolved using Slidebook 5.0 software (Intelligent imaging innovations, Denver, $\mathrm{CO}$ ). Image quantification was performed with imageJ software (NIH). Volume rendering was performed with Slidebook 5.0 software. Any adjustment of brightness or contrast was performed using Slidebook 5.0 software, and always applied to the entire image. 


\section{Western blotting methods}

Third instar larvae were dissected and muscle preparations were immediately transferred into $5 \times$ SDS sample buffer and denatured by boiling for $10 \mathrm{~min}$. Proteins were resolved by SDS-PAGE on a 4-12\% Bis-Tris gel (Life Technologies), transferred to a nitrocellulose membrane, immunoblotted with primary and HRP-conjugated secondary antibodies (Life Technologies) and detected using an ECL chemi-luminescent reagent (Life Technologies). The following primary antibodies were used: anti-VCP (Cell Signaling, Danvers, MA) at 1:1000, anti- GRP78/HSPA5 (Thermo Scientific) at 1:2500, insect antiCathepsin L (R\&D Systems, Minneapolis, MN) at 1:1000, and anti-Tubulin E7-c (Developmental Studies Hybridoma Bank, University of lowa, IA) at 1:10,000. Secondary HRP conjugated antibodies (GE Lifesciences, Pittsburgh, PA) were used at 1:5000.

\section{Electrophysiology methods}

Sharp-electrode recordings were made from muscle 6 in abdominal segments 2 and 3 from thirdinstar larvae using an Axoclamp 900A amplifier (Molecular Devices), as described previously (Frank et al., 2006). Recordings were made in $\mathrm{HL} 3$ saline containing the following components: $\mathrm{NaCl}(70$ $\mathrm{mM}), \mathrm{KCl}(5 \mathrm{mM}), \mathrm{MgCl} 2(10 \mathrm{mM}), \mathrm{NaHCO} 3(10 \mathrm{mM})$, sucrose $(115 \mathrm{mM})$, trehalose $(5 \mathrm{mM})$, HEPES (5 $\mathrm{mM})$, and $\mathrm{CaCl} 2(0.3 \mathrm{mM})$. Mean EPSP, mEPSP amplitude, and Rin were obtained by averaging values across all NMJs for a given genotype. EPSP traces were analyzed with custom-written routines in MATLAB (Mathworks, Natick, MA) as previously described (Gaviño et al., 2015). mEPSP traces were analyzed in IGOR Pro 6.3 (Wave-Metrics; custom script submitted with this manuscript).

\section{Acknowledgements}

We thank members of the Davis lab for helpful advice and critically reading the manuscript. We are grateful to Kevin Ford and Ryan Jones for providing custom scripts for EPSP and mEPSP analyses. We also thank TK Sang and B Ye for generously sharing fly stocks. AEJ is a fellow of the Jane Coffin Childs Memorial Fund for Medical Research. This study was supported by NIH grant NS047342 to GWD.

\section{Additional information}

Competing interests

GWD: Reviewing editor, eLife. The other authors declare that no competing interests exist.

Funding

\begin{tabular}{lll} 
Funder & Grant reference & Author \\
\hline National Institutes of Health & R37NS047342 & Graeme W Davis \\
\hline $\begin{array}{l}\text { Jane Coffin Childs Memorial Fund } \\
\text { for Medical Research }\end{array}$ & Postdoctoral Fellowship & Alyssa E Johnson \\
\hline
\end{tabular}

The funders had no role in study design, data collection and interpretation, or the decision to submit the work for publication.

Author contributions

AEJ, Conception and design, Acquisition of data, Analysis and interpretation of data, Drafting or revising the article; HS, Conception and design, Acquisition of data, Analysis and interpretation of data; AGH, Acquisition of data, Analysis and interpretation of data; AT, Contributed unpublished essential data or reagents; GWD, Conception and design, Drafting or revising the article

\section{References}

Abramzon Y, Johnson JO, Scholz SW, Taylor JP, Brunetti M, Calvo A, Mandrioli J, Benatar M, Mora G, Restagno G, Chiò A, Traynor BJ. 2012. Valosin-containing protein (VCP) mutations in sporadic amyotrophic lateral sclerosis. Neurobiology of Aging 33:2231. doi: 10.1016/j.neurobiolaging.2012.04.005.

Baek GH, Cheng H, Choe V, Bao X, Shao J, Luo S, Rao H. 2013. Cdc48: a swiss army knife of cell biology. Journal of Amino Acids 2013:183421. doi: 10.1155/2013/183421.

Chang YC, Hung WT, Chang YC, Chang HC, Wu CL, Chiang AS, Jackson GR, Sang TK. 2011. Pathogenic VCP/

TER94 alleles are dominant actives and contribute to neurodegeneration by altering cellular ATP level in a Drosophila IBMPFD model. PLOS Genetics 7:e1001288. doi: 10.1371/journal.pgen.1001288. 
Chou TF, Brown SJ, Minond D, Nordin BE, Li K, Jones AC, Chase P, Porubsky PR, Stoltz BM, Schoenen FJ, Patricelli MP, Hodder P, Rosen H, Deshaies RJ. 2011. Reversible inhibitor of p97, DBeQ, impairs both ubiquitin-dependent and autophagic protein clearance pathways. Proceedings of the National Academy of Sciences of USA 108: 4834-4839. doi: 10.1073/pnas.1015312108.

Clemen CS, Tangavelou K, Strucksberg KH, Just S, Gaertner L, Regus-Leidig H, Stumpf M, Reimann J, Coras R, Morgan RO, Fernandez MP, Hofmann A, Müller S, Schoser B, Hanisch FG, Rottbauer W, Blümcke I, von Hörsten S, Eichinger L, Schröder R. 2010. Strumpellin is a novel valosin-containing protein binding partner linking hereditary spastic paraplegia to protein aggregation diseases. Brain 133:2920-2941. doi: 10.1093/brain/awq222.

Cornelissen T, Haddad D, Wauters F, Van Humbeeck C, Mandemakers W, Koentjoro B, Sue C, Gevaert K, De Strooper B, Verstreken P, Vandenberghe W. 2014. The deubiquitinase USP15 antagonizes Parkin-mediated mitochondrial ubiquitination and mitophagy. Human Molecular Genetics 23:5227-5242. doi: 10.1093/hmg/ddu244.

Dargemont C, Ossareh-Nazari B. 2012. Cdc48/p97, a key actor in the interplay between autophagy and ubiquitin/ proteasome catabolic pathways. Biochimica Et Biophysica Acta 1823:138-144. doi: 10.1016/j.bbamcr.2011. 07.011.

Dermaut B, Norga KK, Kania A, Verstreken P, Pan H, Zhou Y, Callaerts P, Bellen HJ. 2005. Aberrant lysosomal carbohydrate storage accompanies endocytic defects and neurodegeneration in Drosophila benchwarmer. The Journal of Cell Biology 170:127-139. doi: 10.1083/jcb.200412001.

Diaper DC, Adachi Y, Lazarou L, Greenstein M, Simoes FA, Di Domenico A, Solomon DA, Lowe S, Alsubaie R, Cheng D, Buckley S, Humphrey DM, Shaw CE, Hirth F. 2013. Drosophila TDP-43 dysfunction in glia and muscle cells cause cytological and behavioural phenotypes that characterize ALS and FTLD. Human Molecular Genetics 22:3883-3893. doi: 10.1093/hmg/ddt243.

Dolan PJ, Jin YN, Hwang W, Johnson GV. 2011. Decreases in valosin-containing protein result in increased levels of tau phosphorylated at Ser262/356. FEBS Letters 585:3424-3429. doi: 10.1016/j.febslet.2011.09.032.

Frank CA, Kennedy MJ, Goold CP, Marek KW, Davis GW. 2006. Mechanisms underlying the rapid induction and sustained expression of synaptic homeostasis. Neuron 52:663-677. doi: 10.1016/j.neuron.2006.09.029.

Gaviño MA, Ford KJ, Archila S, Davis GW. 2015. Homeostatic synaptic depression is achieved through a regulated decrease in presynaptic calcium channel abundance. eLife 4:e05473. doi: 10.7554/eLife.05473.

Gonzalez MA, Feely SM, Speziani F, Strickland AV, Danzi M, Bacon C, Lee Y, Chou TF, Blanton SH, Weihl CC, Zuchner S, Shy ME. 2014. A novel mutation in VCP causes Charcot-Marie-Tooth type 2 disease. Brain 137: 2897-2902. doi: 10.1093/brain/awu224.

Halawani D, Latterich M. 2006. p97: the cell's molecular purgatory? Molecular Cell 22:713-717. doi: 10.1016/j. molcel.2006.06.003.

Huotari J, Helenius A. 2011. Endosome maturation. The EMBO Journal 30:3481-3500. doi: 10.1038/emboj. 2011.286.

Huss M, Ingenhorst G, König S, Gassel M, Dröse S, Zeeck A, Altendorf K, Wieczorek H. 2002. Concanamycin A, the specific inhibitor of V-ATPases, binds to the V(o) subunit c. The Journal of Biological Chemistry 277:40544-40548. doi: 10.1074/jbc.M207345200.

Ju JS, Weihl CC. 2010. Inclusion body myopathy, Paget's disease of the bone and fronto-temporal dementia: a disorder of autophagy. Human Molecular Genetics 19:R38-R45. doi: 10.1093/hmg/ddq157.

Ju JS, Fuentealba RA, Miller SE, Jackson E, Piwnica-Worms D, Baloh RH, Weihl CC. 2009. Valosin-containing protein (VCP) is required for autophagy and is disrupted in VCP disease. The Journal of Cell Biology 187: 875-888. doi: 10.1083/jcb.200908115.

Kim NC, Tresse E, Kolaitis RM, Molliex A, Thomas RE, Alami NH, Wang B, Joshi A, Smith RB, Ritson GP, Winborn BJ, Moore J, Lee JY, Yao TP, Pallanck L, Kundu M, Taylor JP. 2013. VCP is essential for mitochondrial quality control by PINK1/Parkin and this function is impaired by VCP mutations. Neuron 78:65-80. doi: 10.1016/j.neuron. 2013.02.029.

Kimonis VE, Mehta SG, Fulchiero EC, Thomasova D, Pasquali M, Boycott K, Neilan EG, Kartashov A, Forman MS, Tucker S, Kimonis K, Mumm S, Whyte MP, Smith CD, Watts GD. 2008. Clinical studies in familial VCP myopathy associated with Paget disease of bone and frontotemporal dementia. American Journal of Medical Genetics. Part A 146A:745-757. doi: 10.1002/ajmg.a.31862.

Knapp PE, Swanson JA. 1990. Plasticity of the tubular lysosomal compartment in macrophages. Journal of Cell Science 95:433-439.

Latterich M, Fröhlich KU, Schekman R. 1995. Membrane fusion and the cell cycle: Cdc48p participates in the fusion of ER membranes. Cell 82:885-893. doi: 10.1016/0092-8674(95)90268-6.

Liewluck T, Milone M, Mauermann ML, Castro-Couch M, Cerhan JH, Murthy NS. 2014. A novel VCP mutation underlies scapuloperoneal muscular dystrophy and dropped head syndrome featuring lobulated fibers. Muscle Nerve 50:295-299. doi: 10.1002/mus.24290.

Masiero E, Agatea L, Mammucari C, Blaauw B, Loro E, Komatsu M, Metzger D, Reggiani C, Schiaffino S, Sandri M. 2009. Autophagy is required to maintain muscle mass. Cell Metabolism 10:507-515. doi: 10.1016/j.cmet.2009. 10.008.

Meyer H, Bug M, Bremer S. 2012. Emerging functions of the VCP/p97 AAA-ATPase in the ubiquitin system. Nature Cell Biology 14:117-123. doi: 10.1038/ncb2407.

Mizushima N, Yamamoto A, Matsui M, Yoshimori T, Ohsumi Y. 2004. In vivo analysis of autophagy in response to nutrient starvation using transgenic mice expressing a fluorescent autophagosome marker. Molecular Biology of the Cell 15:1101-1111. doi: 10.1091/mbc.E03-09-0704.

Moir D, Stewart SE, Osmond BC, Botstein D. 1982. Cold-sensitive cell-division-cycle mutants of yeast: isolation, properties, and pseudoreversion studies. Genetics 100:547-563. 
Nezis IP, Shravage BV, Sagona AP, Lamark T, Bjørkøy G, Johansen T, Rusten TE, Brech A, Baehrecke EH, Stenmark H. 2010. Autophagic degradation of dBruce controls DNA fragmentation in nurse cells during late Drosophila melanogaster oogenesis. The Journal of Cell Biology 190:523-531. doi: 10.1083/jcb.201002035.

Piccirillo R, Goldberg AL. 2012. The p97/VCP ATPase is critical in muscle atrophy and the accelerated degradation of muscle proteins. The EMBO Journal 31:3334-3350. doi: 10.1038/emboj.2012.178.

Ralston E, Ploug T, Kalhovde J, Lomo T. 2001. Golgi complex, endoplasmic reticulum exit sites, and microtubules in skeletal muscle fibers are organized by patterned activity. The Journal of Neuroscience 21:875-883.

Ren C, Finkel SE, Tower J. 2009. Conditional inhibition of autophagy genes in adult Drosophila impairs immunity without compromising longevity. Experimental Gerontology 44:228-235. doi: 10.1016/j.exger.2008.10.002.

Rong Y, McPhee CK, McPhee C, Deng S, Huang L, Chen L, Liu M, Tracy K, Baehrecke EH, Baehreck EH, Yu L, Lenardo MJ. 2011. Spinster is required for autophagic lysosome reformation and mTOR reactivation following starvation. Proceedings of the National Academy of Sciences of USA 108:7826-7831. doi: 10.1073/pnas. 1013800108.

Rong Y, Liu M, Ma L, Du W, Zhang H, Tian Y, Cao Z, Li Y, Ren H, Zhang C, Li L, Chen S, Xi J, Yu L. 2012. Clathrin and phosphatidylinositol-4,5-bisphosphate regulate autophagic lysosome reformation. Nature Cell Biology 14: 924-934. doi: 10.1038/ncb2557.

Sandri M. 2010. Autophagy in skeletal muscle. FEBS Letters 584:1411-1416. doi: 10.1016/j.febslet.2010.01.056.

Swanson J, Burke E, Silverstein SC. 1987a. Tubular lysosomes accompany stimulated pinocytosis in macrophages. The Journal of Cell Biology 104:1217-1222. doi: 10.1083/jcb.104.5.1217.

Swanson J, Bushnell A, Silverstein SC. 1987b. Tubular lysosome morphology and distribution within macrophages depend on the integrity of cytoplasmic microtubules. Proceedings of the National Academy of Sciences of USA 84:1921-1925. doi: 10.1073/pnas.84.7.1921.

Sweeney ST, Davis GW. 2002. Unrestricted synaptic growth in spinster-a late endosomal protein implicated in TGF-beta-mediated synaptic growth regulation. Neuron 36:403-416. doi: 10.1016/S0896-6273(02)01014-0.

Szweda PA, Camouse M, Lundberg KC, Oberley TD, Szweda LI. 2003. Aging, lipofuscin formation, and free radical-mediated inhibition of cellular proteolytic systems. Ageing Research Reviews 2:383-405. doi: 10.1016/ S1568-1637(03)00028-X.

Taylor EB, Rutter J. 2011. Mitochondrial quality control by the ubiquitin-proteasome system. Biochemical Society Transactions 39:1509-1513. doi: 10.1042/BST0391509.

Tresse E, Salomons FA, Vesa J, Bott LC, Kimonis V, Yao TP, Dantuma NP, Taylor JP. 2010a. VCP/p97 is essential for maturation of ubiquitin-containing autophagosomes and this function is impaired by mutations that cause IBMPFD. Autophagy 6:217-227. doi: 10.4161/auto.6.2.11014.

Tresse E, Salomons FA, Vesa J, Bott LC, Kimonis V, Yao TP, Dantuma NP, Taylor JP. 2010b. VCP/p97 is essential for maturation of ubiquitin-containing autophagosomes and this function is impaired by mutations that cause IBMPFD. Autophagy 6:217-227. doi: 10.4161/auto.6.2.11014.

Vesa J, Su H, Watts GD, Krause S, Walter MC, Martin B, Smith C, Wallace DC, Kimonis VE. 2009. Valosin containing protein associated inclusion body myopathy: abnormal vacuolization, autophagy and cell fusion in myoblasts. Neuromuscular Disorders 19:766-772. doi: 10.1016/j.nmd.2009.08.003.

Wang Y, Ballar P, Zhong Y, Zhang X, Liu C, Zhang YJ, Monteiro MJ, Li J, Fang S. 2011. SVIP induces localization of p97/NCP to the plasma and lysosomal membranes and regulates autophagy. PLOS ONE 6:e24478. doi: 10.1371/ journal.pone.0024478.

Watts GD, Wymer J, Kovach MJ, Mehta SG, Mumm S, Darvish D, Pestronk A, Whyte MP, Kimonis VE. 2004. Inclusion body myopathy associated with Paget disease of bone and frontotemporal dementia is caused by mutant valosin-containing protein. Nature Genetics 36:377-381. doi: 10.1038/ng1332.

Weihl CC, Pestronk A, Kimonis VE. 2009. Valosin-containing protein disease: inclusion body myopathy with Paget's disease of the bone and fronto-temporal dementia. Neuromuscular Disorders 19:308-315. doi: 10.1016/j.nmd. 2009.01.009.

Wójcik C, Rowicka M, Kudlicki A, Nowis D, McConnell E, Kujawa M, DeMartino GN. 2006. Valosin-containing protein (p97) is a regulator of endoplasmic reticulum stress and of the degradation of $\mathrm{N}$-end rule and ubiquitinfusion degradation pathway substrates in mammalian cells. Molecular Biology of the Cell 17:4606-4618.

Wolf DH, Stolz A. 2012. The Cdc48 machine in endoplasmic reticulum associated protein degradation. Biochimica Et Biophysica Acta 1823:117-124. doi: 10.1016/j.bbamcr.2011.09.002.

Xie Z, Klionsky DJ. 2007. Autophagosome formation: core machinery and adaptations. Nature Cell Biology 9 : 1102-1109. doi: 10.1038/ncb1007-1102.

Yu L, McPhee CK, Zheng L, Mardones GA, Rong Y, Peng J, Mi N, Zhao Y, Liu Z, Wan F, Hailey DW, Oorschot V, Klumperman J, Baehrecke EH, Lenardo MJ. 2010. Termination of autophagy and reformation of lysosomes regulated by mTOR. Nature 465:942-946. doi: 10.1038/nature09076. 